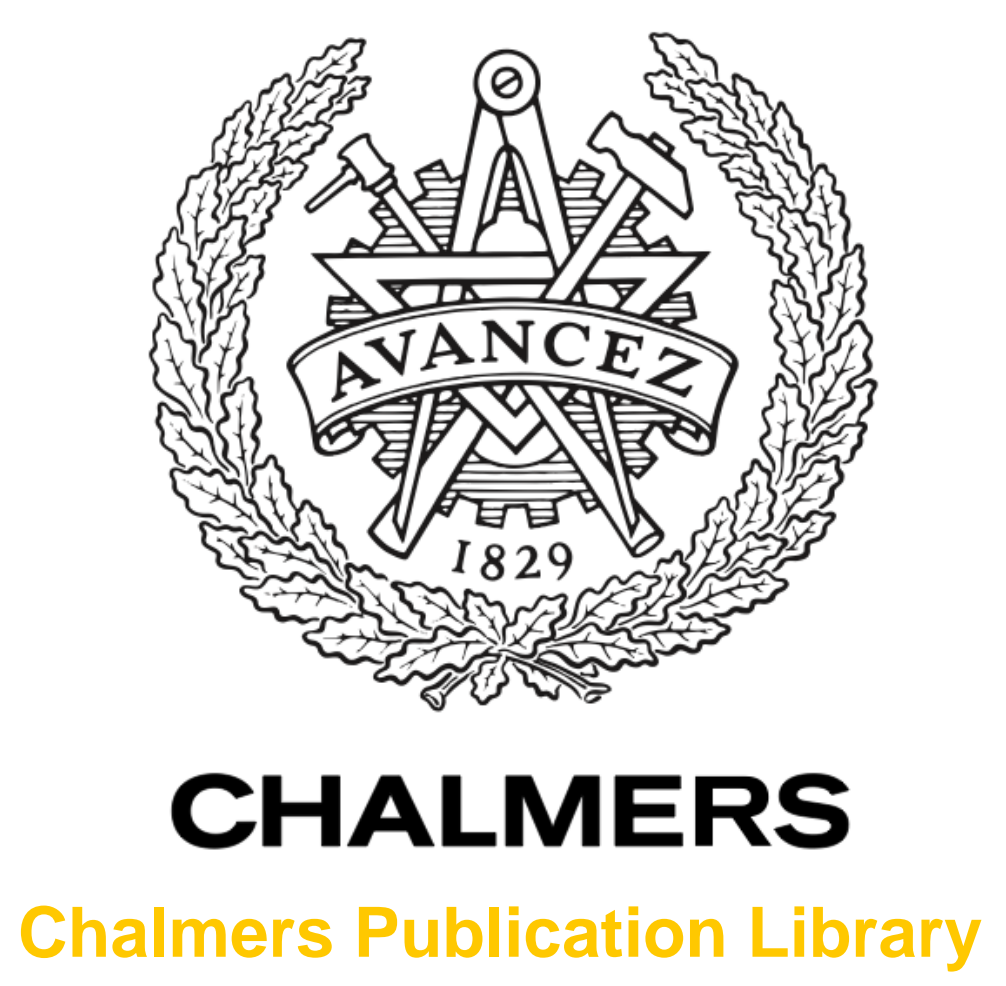

\title{
Traffic-adaptive Signal Control and Vehicle Routing Using a Decentralized Back- pressure Method
}

This document has been downloaded from Chalmers Publication Library (CPL). It is the author's version of a work that was accepted for publication in:

In proceedings of European Control Conference, ECC'15, July 15-17, Linz, Austria

Citation for the published paper:

Zaidi, A. ; Kulcsar, B. ; Wymeersch, H. (2015) "Traffic-adaptive Signal Control and Vehicle Routing Using a Decentralized Back-pressure Method". In proceedings of European Control Conference, ECC'15, July 15-17, Linz, Austria

Downloaded from: http://publications.lib.chalmers.se/publication/216049

Notice: Changes introduced as a result of publishing processes such as copy-editing and formatting may not be reflected in this document. For a definitive version of this work, please refer to the published source. Please note that access to the published version might require a subscription. 


\title{
Traffic-adaptive Signal Control and Vehicle Routing Using a Decentralized Back-pressure Method
}

\author{
Ali A. Zaidi, Balázs Kulcsár, and Henk Wymeersch
}

\begin{abstract}
The problem of controlling traffic lights under adaptive routing of vehicles in urban road networks is considered. Multi-commodity back-pressure algorithms, originally developed for routing and scheduling in communication networks, are applied to road networks to control traffic lights and adaptively reroute vehicles. The performance of the algorithms is analyzed using a microscopic traffic simulator. The results demonstrate that the proposed signal control and adaptive routing algorithms can provide significant improvement over a fixed schedule and a single-commodity back-pressure signal controller, in terms of various performance metrics, including queue-length, trips completed, and travel times.
\end{abstract}

\section{INTRODUCTION}

Recent technological developments in sensing, communication, and low-power electronics, allow real-time measurement, availability, and processing of traffic data, thus providing an opportunity to deploy traffic-adaptive intelligent signal controllers. An efficient implementation of trafficadaptive signal control methods based on real-time traffic measurements can improve conditions for both the drivers and the environment, in terms of travel times, fuel consumption, pollution, and accidents [1]. Traditionally, traffic light controllers follow a pre-defined optimized schedule [2], which may result in a poor performance under time-varying traffic conditions and under very high traffic demands. This problem can be alleviated through adaptive traffic signal controllers, such as SCOOT, UTOPIA, SCATS, or RHODES [3]-[5]. In these adaptive traffic controllers, real-time measurements are collected using on-road detectors. Based on these measurements, either the parameters (splits, offsets, cycle-length) of the signal plans are adjusted on a cycleto-cycle basis or a best signal plan is selected from a predefined set of signal plans. The implementation of these methods, however, requires centralized decision making for all intersections based on the traffic related measurements. In addition to these traffic-adaptive signal control implementations, other centralized traffic signal control algorithms have recently been proposed [6]-[9] using different approaches from control theory such as linear quadratic regulator, robust control, and model predictive control.

Ali A. Zaidi is with Radio Access Technologies, Ericsson Research, Stockholm, Sweden. Email: ali.zaidi@ericsson.com

Balázs Kulcsár and Henk Wymeersch are with the Department of Signals \& Systems, Chalmers University of Technology, Gothenburg, Sweden. Emails: \{kulcsar,henkw\}@ chalmers.se.

This research is supported by SAFER grant on "Enhanced traffic flow optimisation" and the Transport Area of Advance at Chalmers University of Technology. It is also supported, in part, by the European Research Council under Grant No. 258418 (COOPNET).
In contrast to the many centralized approaches for traffic signal control, the literature on decentralized solutions, which would be very useful especially for large urban areas, is scarce. Recently, researchers in the transportation and control communities have proposed different trafficadaptive scalable and distributed methods [10]-[14], where the general idea is to solve a separate optimization problem for every intersection. These per-intersection optimization problems are loosely coupled via real-time traffic conditions. The implementation of these controllers requires either the knowledge of expected traffic load on the links associated with the intersection during the next cycle, or the difference between the traffic loads on the links associated with the network. Many of these schemes are inspired by scheduling and routing algorithms in wireless networks, in particular the well-known back-pressure scheme from [15]. Back-pressure is a decentralized scheme the can provide maximum network throughput under the assumptions that all links in the network have infinite capacities (it is in fact optimal in the sense of supporting maximum traffic arrival rates that guarantee stability of queues in a stochastic sense). This idea was first adapted to urban road networks in [11], where it was shown that significant performance gains can be achieved in terms of network queue lengths by employing a back-pressure scheme for signal control. It was also shown to provide good performance compared to the fixed time schedule controllers, when the links have finite capacities. However, [11] does not dynamically re-route vehicles, leading to local bottlenecks.

In this paper, we extend [11] by performing both trafficadaptive signal control and routing, under back-pressure based control methods. In particular, rather than a single commodity back-pressure scheme with fixed routes as in [11], we apply a multi-commodity (one commodity per destination) version of the back-pressure scheme under adaptive route selection [16]. Our results demonstrate that the proposed schemes can provide significant performance gains.

\section{Problem Formulation}

\section{A. Road Network}

Consider an urban road network comprised of $N$ links/roads and $L$ junctions (signalized intersections). We model the network as a directed graph $\mathcal{G}=(\mathcal{R}, \mathcal{J})$, where $\mathcal{R}=\left\{R_{1}, R_{2}, \ldots, R_{N}\right\}$ is the set of links and $\mathcal{J}=$ $\left\{J_{1}, J_{2}, \ldots, J_{L}\right\}$ is the set of junctions in the road network. A vehicle exogenously enters the network from a certain link (origin), travels along one or more links in the network and finally leaves the network at a certain link (destination). Thus, for each vehicle in the network, there is an associated 


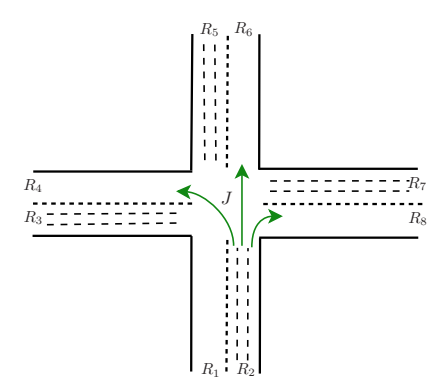

Fig. 1. A four-way junction with 8 roads and 12 possible traffic movements.
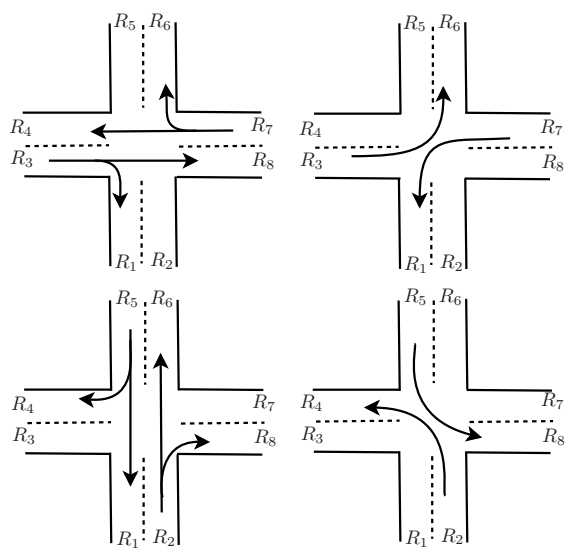

Fig. 2. Typical phases through a four-way junction.

origin and destination pair. All vehicles that have a common origin and destination pair constitute a flow $f$. Let $\mathcal{F}$ be the set of all flows in the network and let $(o(f), d(f))$ be the origin-destination pair for a flow $f \in \mathcal{F}$, where $o(f), d(f) \in \mathcal{R}$. Let $\lambda_{f}(t)$ be the rate at which vehicles associated with flow $f$ exogenously enter $o(f)$ at discrete time slots $t \in \mathbb{N}$, with $\lim _{T \rightarrow \infty} \frac{1}{T} \sum_{t=1}^{T} \mathbb{E}\left[\lambda_{f}(t)\right]=\lambda_{f}$. We assume that the flow arrival processes are independent of each other and also independent across time slots and have finite second moments. At any time $t$, let $Q_{a b}(t)$ be the number of vehicles queued in a link $R_{a}$ to move to an adjacent link $R_{b}$ and let $Q_{a}(t)=\sum_{b} Q_{a b}(t)$ be the the total queue length at link $R_{a}$.

\section{B. Traffic Signal Control}

Each junction has certain traffic movements associated with it. A traffic movement through a junction corresponding to the vehicles exiting $R_{a}$ and entering $R_{b}$ is denoted by the pair $\left(R_{a}, R_{b}\right)$. Let $\mathcal{M}_{i}$ be the set of all traffic movements through a junction $J_{i}$. Consider an example of a four-way junction in Fig. 1, where there are twelve possible traffic movements. The set of all possible traffic movements for this four-way junction is given by

$$
\begin{aligned}
\mathcal{M}=\{ & \left(R_{3}, R_{8}\right),\left(R_{3}, R_{1}\right),\left(R_{7}, R_{4}\right),\left(R_{7}, R_{6}\right), \\
& \left(R_{3}, R_{6}\right),\left(R_{7}, R_{1}\right),\left(R_{5}, R_{4}\right),\left(R_{5}, R_{1}\right), \\
& \left.\left(R_{2}, R_{6}\right),\left(R_{2}, R_{8}\right),\left(R_{2}, R_{4}\right),\left(R_{5}, R_{8}\right)\right\} .
\end{aligned}
$$

A subset of traffic movements that can occur simultaneously through a junction constitute a phase. Let $\mathcal{P}_{i}=$ $\left\{p_{1}^{i}, p_{2}^{i}, \ldots, p_{l}^{i}\right\}$ be the set of all possible phases through a junction $J_{i}$. As an example, consider a four-way junction in Fig. 2 that has four possible phases given by

$$
\begin{aligned}
& p_{1}=\left\{\left(R_{3}, R_{8}\right),\left(R_{3}, R_{1}\right),\left(R_{7}, R_{4}\right),\left(R_{7}, R_{6}\right)\right\}, \\
& p_{2}=\left\{\left(R_{3}, R_{6}\right),\left(R_{7}, R_{1}\right)\right\}, \\
& p_{3}=\left\{\left(R_{5}, R_{4}\right),\left(R_{5}, R_{1}\right),\left(R_{2}, R_{6}\right),\left(R_{2}, R_{8}\right)\right\}, \\
& p_{4}=\left\{\left(R_{2}, R_{4}\right),\left(R_{5}, R_{8}\right)\right\} .
\end{aligned}
$$

Furthermore, we assume that with every possible movement $\left(R_{a}, R_{b}\right)$ through a junction, there is a rate $s_{a b}(t)$ (the number of vehicles per discrete time unit) with which vehicles flow. For the sake of simplicity, we assume that $s_{a b}(t)=s_{a b}$ for all $t$, i.e., flow rate through a junction does not depend on time or any other state in the road network. However, the control schemes presented in the paper are also applicable to the situations where traffic movement rates are time varying.

\section{Routing of Vehicles}

We consider that each vehicle that enters the network has a fixed destination but the route that it takes towards the destination is dynamically updated based on the traffic conditions. Thus, the vehicles with a common origin and destination pair may not necessarily follow the same route. We consider that for every vehicle, the route is dynamically updated at every junction. Whenever a vehicle enters a link $R_{a}$, its next movement $\left(R_{a}, R_{b}\right)$ through the upcoming junction is decided in real-time and the vehicle joins one of the possible queues (lanes) accordingly. For instance, in the example shown in Fig. 1, when a vehicle enters $R_{2}$, it can join one of the three possible vehicle queues $\left\{Q_{24}, Q_{26}, Q_{28}\right\}$.

\section{Control Problem}

At every junction $J_{i}$, there is a controller $\mathcal{C}_{i}$ that has to perform the following tasks at every time slot $t$.

1) Select a phase $p_{k}^{i}(t) \in \mathcal{P}_{i}$ (i.e., the traffic controller gives the right of way to certain traffic movements in every time slot).

2) Make a routing decision for the vehicles (i.e., assign queues to the vehicles) related to every flow passing through the given junction.

The routing decisions are communicated to the corresponding vehicles and the vehicles in the network are assumed to follow the routing decision made by the traffic controller.

\section{Proposed Methods}

In [11], a back-pressure scheduling algorithm was used for traffic signal control, assuming all vehicles follow fixed routes but ignoring the fact that all vehicles in the network have different destinations (single-commodity back pressure scheme). We now propose to employ multi-commodity backpressure scheme for traffic signal control with adaptive routing of vehicles. In contrast to the single-commodity scheme where the vehicle queue length information has to be known on per-link basis, the operation of multi-commodity schemes require queue length information on per-destination 
basis. Since the numbers of destinations in a road network is normally very big, it is not possible to maintain nonoverlapping vehicle queues on per-destination basis due to the physical constraints. In order to tackle this issue, we utilize the concept of virtual queues, which is essential for the operation of the proposed multi-commodity back-pressure traffic control scheme in road networks.

\section{A. Virtual Queues and Virtual Vehicles}

Following the wireless networking approach in [16], we introduce virtual traffic and virtual queues (referred to as shadow queues in [16]) in the road network. For each vehicle that exogenously enters a link in the network, we generate a virtual vehicle with probability one and another virtual vehicle with probability $\epsilon>0$. Hence, for any flow $f$ in the network, the arrival rate of virtual traffic is $(1+\epsilon) \lambda_{f}(t)$. The reason for introducing $\epsilon$ is discussed in Section III-C.1. With the virtual traffic we can associate virtual queues. We denote the number of virtual vehicles for destination $d$ on link $R_{a}$ by $\tilde{Q}_{a}^{d}$. We note that virtual traffic and queues are merely counters, which form a fictitious queuing system on which the signal control and route control algorithms are based. The real queues $Q_{a b}(t)$ containing real vehicles are maintained on a per movement basis, for every possible movement $\left(R_{a}, R_{b}\right)$ through a given junction.

\section{B. Signal Control Algorithm with Adaptive Routing}

We follow [16], where an algorithm is proposed that decouples routing and scheduling in wireless networks. Adaptive routing operates by placing incoming vehicles in real queues according to a probabilistic routing, whereas signal control is based on a back-pressure method using virtual queues per destination.

1) Signal Control Algorithm: The signal control algorithm for each junction is decentralized. At each junction $J_{i}$, the algorithm works based on the per destination virtual queue length information $\tilde{Q}_{a}^{d}$ for all links $R_{a}$ associated with the given junction. The algorithm works as follows for each junction $J_{i}$ :

1) For all $(a, b)$ such that $\left(R_{a}, R_{b}\right) \in \mathcal{M}_{i}$, determine the destination with maximum back-pressure and then assign a weight to that destination:

$$
\begin{aligned}
d_{a b}^{\star}(t) & =\arg \max _{d}\left\{\tilde{Q}_{a}^{d}(t)-\tilde{Q}_{b}^{d}(t)\right\}, \\
W_{a b}(t) & =\max \left\{\tilde{Q}_{a}^{d_{a b}^{\star}(t)}(t)-\tilde{Q}_{b}^{d_{a b}^{\star}(t)}(t), 0\right\} .
\end{aligned}
$$

2) For each phase $p_{k}^{i} \in \mathcal{P}_{i}$, compute the throughput as

$$
S_{p_{k}^{i}}(t)=\sum_{\left(R_{a}, R_{b}\right) \in p_{k}^{i}} W_{a b}(t) s_{a b}(t) .
$$

3) The controller $C_{i}$ at junction $J_{i}$ activates the phase with the highest pressure, i.e., it selects

$$
p_{k^{\star}}^{i}=\arg \max _{p_{k}^{i} \in \mathcal{P}_{i}} S_{p_{k}^{i}}(t) .
$$

When a certain phase is activated, the real vehicles in the network move according to the given saturation rates and the queues of real vehicles evolve accordingly. The virtual queues evolve according to:

$$
\begin{aligned}
\tilde{Q}_{a}^{d}(t+1) & =\tilde{Q}_{a}^{d}(t)-\sum_{b:\left(R_{a}, R_{b}\right) \in \mathcal{M}_{i}} I_{\left\{d_{a b}^{\star}(t)=d\right\}} s_{a b}(t) \\
& +\sum_{c:\left(R_{c}, R_{a}\right) \in \mathcal{M}_{i}} I_{\left\{d_{c a}^{\star}(t)=d\right\}} s_{c a}(t) \\
& +\sum_{f \in \mathcal{F}} I_{\{o(f)=a, d(f)=d\}} \tilde{\lambda}_{f}(t),
\end{aligned}
$$

where $I_{\{\cdot\}}$ denotes the indicator function and $\tilde{\lambda}_{f}(t)$ is the number of virtual vehicles associated with flow $f$ that exogenously enter $o(f)$ at time $t$.

2) Adaptive Route Control Algorithm: Let $\sigma_{a b}^{d}(t)$ be the number of virtual vehicles transferred from $R_{a}$ to $R_{b}$ for destination $d$ at time $t, \bar{\sigma}_{a b}^{d}$ its expected value in stationary regime, and $\hat{\sigma}_{a b}^{d}(t)$ the estimate of $\bar{\sigma}_{a b}^{d}$ at time $t$.

1) At every junction, compute $\hat{\sigma}_{a b}^{d}(t)$ for every feasible movement $\left(R_{a}, R_{b}\right) \in \mathcal{M}_{i}$ associated with that junction using an exponential averaging method:

$$
\hat{\sigma}_{a b}^{d}(t)=(1-\beta) \hat{\sigma}_{a b}^{d}(t-1)+\beta \sigma_{a b}^{d}(t),
$$

where $0<\beta<1$ is a smoothing factor.

2) Compute the routing probabilities:

$$
P_{a b}^{d}(t)=\frac{\hat{\sigma}_{a b}^{d}(t)}{\sum_{c:\left(R_{a}, R_{c}\right) \in \mathcal{M}_{i}} \hat{\sigma}_{a c}^{d}(t)} .
$$

3) A vehicle entering link $R_{a}$ joins real queue $Q_{a b}$ with probability $P_{a b}^{d}(t)$. That is, the vehicle entering $R_{a}$ destined for $R_{d}$ will be routed to $R_{b}$ with probability $P_{a b}^{d}(t)$ at time $t$ through the junction $J_{i}$.

The routing information is communicated to vehicles in terms of probabilities or percentages. For example, consider that for the four-way junction illustrated in Fig. 1 if $\left[P_{24}^{d}(t)=\right.$ $\left.0.1, P_{26}^{d}(t)=0.2, P_{28}^{d}(t)=0.7\right]$, then among all those vehicles that enter link 2 having destination $d$, 10 percent should join queue $Q_{24}, 20$ percent should join queue $Q_{26}$, and 70 percent should join queue $Q_{28}$.

\section{Stability and Optimality}

1) Infinite Length Links: In this section we discuss optimality of the proposed methods in the sense of supporting maximum traffic arrivals in a road network under the assumption that all links are infinitely long. To be precise, we first define stochastic stability and capacity region of a network and then discuss the optimality.

If the route of every flow is fixed, then the capacity region is the set of all flow arrivals that are supportable given the set of flows and their corresponding routes. We note that the capacity region of a network under fixed flow routes cannot be larger than the capacity of the same network where routes of the flows are not fixed, since capacity region cannot decrease by removing path constraints on flows.

The traffic control and routing algorithms proposed above are based on the algorithms for scheduling (rate allocation) and routing of packets in a communication network. This algorithm is throughput-optimal according to [16, Theorem 


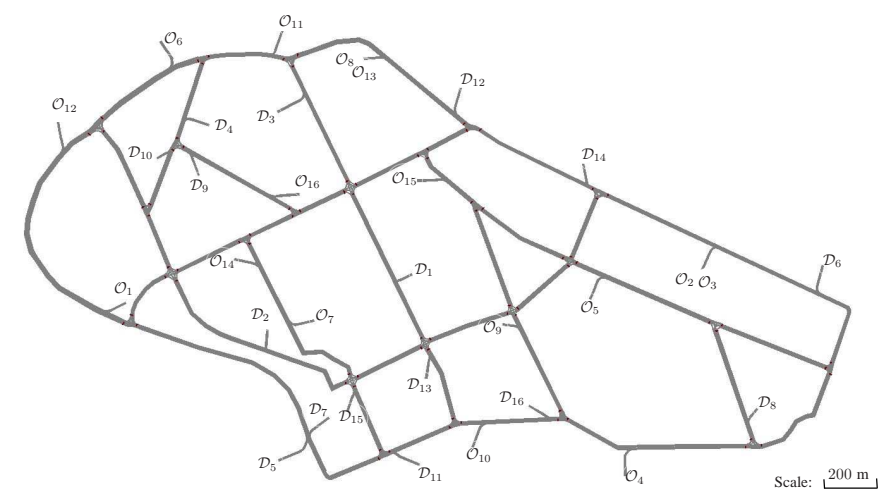

Fig. 3. Road network with 24 intersections, 84 links, 16 origins and 16 destinations.

1], where the authors show that if a network is stable under the back-pressure scheme based on real queues (which is already known to be optimal) with flow arrival rate $\lambda_{f}$, then it is also stable (i.e., real queues are stable) under the back-pressure scheme based on virtual queues with flow arrival rate $\lambda_{f}(1+\epsilon)$, for all $\epsilon>0$. In the proposed traffic control algorithm, the phase activation (activation of a set of movements) procedure is equivalent to scheduling over a communication network. In contrast to a wireless communication network, this scheduling procedure is decentralized at every junction because the activation of links associated with one junction does not affect the activation of links at any other junction if one assumes links to be infinitely long. The possible traffic movements associated with a given junction can be interpreted as connections in a wireless network. Similar to a wireless network where packets cannot be simultaneously transmitted with high rates over neighboring links due to cross-link interference, here in the road network, some movements at a junction cannot be activated simultaneously. With this interpretation and equivalence in mind, we establish optimality of the proposed traffic control and routing algorithms. The algorithms are optimal in the sense that they can stably support any flow arrival rate which is in the interior of the capacity region.

2) Finite Length Links: When a network has links with finite lengths, the issue of stability does not arise because the queues can never be unstable due to finite length links. In this situation, stability corresponds to maintaining bounded queue backlogs in the links where traffic is being input to the network (ingress buffers in the context of communication networks [17]), assuming that origin links can be infinitely long. It is not known if the back-pressure based schemes are throughput-optimal in this context. In the following section, we analyze performance of the proposed back-pressure based algorithms over a network having links of finite lengths.

\section{Performance Analysis}

We analyze performance of the proposed algorithms in terms of queue lengths, trips completed, and travel time using PTV VISSIM [18], which is a microscopic traffic simulator. Within VISSIM, every vehicle is simulated individually and several useful properties related to every vehicle can be accessed dynamically. We will consider and compare three distinct methods:

- Fixed time (FT) schedule signal controller: The possible phases at each intersection are activated in a predetermined periodic fashion. All vehicles are assumed to follow shortest routes to their respective destinations.

- Single-commodity back-pressure (SC-BP) controller: As proposed in [11], each junction $i$ maintain queues $Q_{a}(t)$ for all connected links. For each pair $\left(R_{a}, R_{b}\right) \in \mathcal{M}_{i}$, the back pressure $W_{a b}(t)=Q_{a}(t)-Q_{b}(t)$ is computed. For each phase $p_{k}^{i} \in \mathcal{P}_{i}$, the pressure release is computed as $S_{p_{k}^{i}}(t)=\sum_{\left(R_{a}, R_{b}\right) \in p_{k}^{i}} W_{a b}(t) s_{a b}(t)$. Finally, the phase giving rise to the maximum pressure release is selected. All vehicles are assumed to follow shortest routes to their respective destinations.

- Adaptive routing back-pressure control (AR-BP): The method described in Section III-B.

\section{A. Network and Simulation Parameters}

The simulations are performed using a road network from a central region in the Stockholm area, comprising 24 signalized intersections (16 three-way intersections and 8 four-way intersections) and 84 links. The network is depicted in Fig. 3. The lengths of the longest and the shortest links are approximately 1980 meters and 333 meters. All links are assumed to have three lanes, where each lane is 3.5 meters wide. There are 16 traffic origins $\left\{\mathcal{O}_{1}, \mathcal{O}_{2}, \ldots, \mathcal{O}_{16}\right\}$ and 16 destinations $\left\{\mathcal{D}_{1}, \mathcal{D}_{2}, \ldots, \mathcal{D}_{16}\right\}$ in the network. The traffic associated with an origin-destination pair $\mathcal{O}_{i}-\mathcal{D}_{i}$ forms a flow $f_{i}$. Hence, there are 16 traffic flows in total, $\left\{f_{1}, f_{2}, \ldots, f_{16}\right\}$. We perform simulations with cars of dimensions 4.11 meters $\times 1.5$ meters and 4.76 meters $\times 1.5$ meters. The maximum speed of all vehicles is set to $70 \mathrm{~km} / \mathrm{h}$, as some of the links on the boundary of the network in Fig. 3 are highways. We assume that a car is in a queue if its speed is below a certain threshold (here set to $5 \mathrm{~km} / \mathrm{h}$ ). For the fixed time schedule control (FT), we assume the time period of each cycle equal to 60 seconds at all intersections (both three-way and four-way) according to the signal plan 


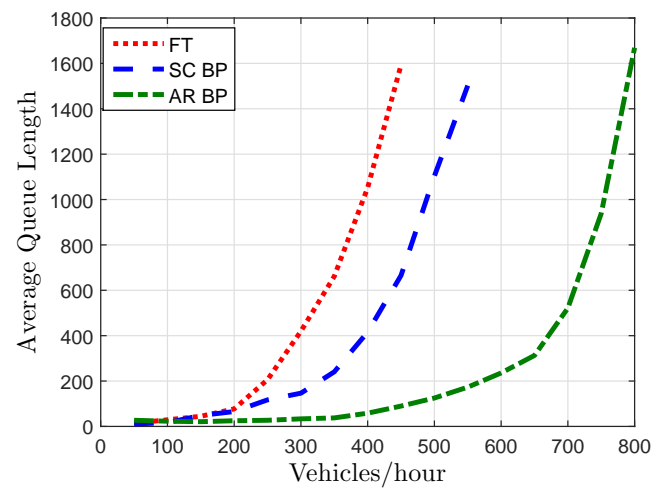

Fig. 4. Average queue lengths over a 2 hours long simulation time period as a function of the vehicle input arrival rate under different control methods.

(phase distribution) given in Table $\mathrm{I}^{1}$. For the back-pressure methods, we consider that a phase is activated after every 15 seconds. All simulations are performed for 7200 simulation seconds (i.e., 2 hours). Within VISSIM, we have set the simulation speed to 10 simulation seconds per second and the simulation resolution is set equal to 1 in order to generate fastest simulation results. Moreover, all the traffic related measurements are taken after every 15 simulation seconds and the signal phase selections and the routing decisions are accordingly updated.

TABLE I

Phase Distributions

\begin{tabular}{|c|c|c|c|c|}
\hline Intersection Type & $p_{1}$ & $p_{2}$ & $p_{3}$ & $p_{4}$ \\
\hline \hline Four-way & 18 sec. & 12 sec. & 18 sec. & 12 sec. \\
\hline Three-way & 24 sec. & 12 sec. & 24 sec. & - \\
\hline
\end{tabular}

\section{B. Simulation Results and Discussions}

In Fig. 4 and Fig. 5 we plot the average queue lengths and average travel times of vehicles from their origins to their respective destinations as functions of vehicle arrival rates under different control methods. Here, an arrival rate of $x$ vehicles/hour per origin means that approximately $16 x$ vehicles enter the network per hour since there are 16 origins. The averages are taken over simulation time in the case of queue length and over both simulation time and number of vehicles in the case of travel time. It was already shown in [11] that SC-BP outperforms FT in terms of queue lengths. An additional observation according to Figs. 4-5 is that AR$\mathrm{BP}$ has the potential to provide considerable improvement over the single-commodity scheme. The behavior of AR-BP is not straightforward - it provides relatively much smaller queue lengths but the travel times are very high at low traffic volumes. Normally, one expects that a larger queue length should lead to a higher travel time. In order to study what makes the average travel times so high under AR$\mathrm{BP}$, we must consider the average speed of vehicles under all schemes. Fig. 6 shows that average vehicle speeds are

\footnotetext{
${ }^{1}$ The time-loss due to amber or yellow signals is not considered in the simulations, however, it can be incorporated easily in VISSIM.
}

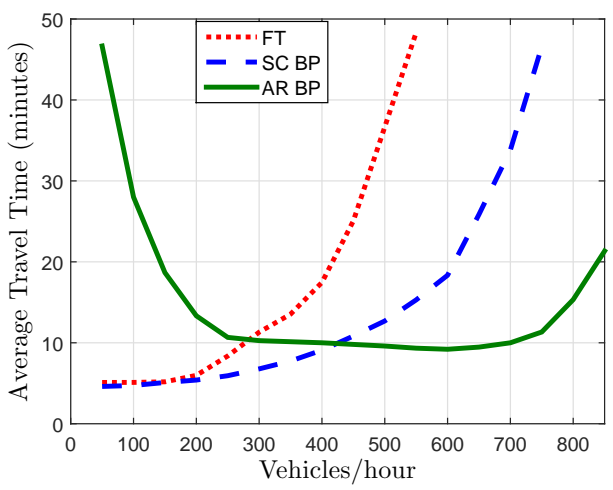

Fig. 5. Average travel times computed over a 2 hours simulation period as a function of the vehicle input arrival rate under different methods.

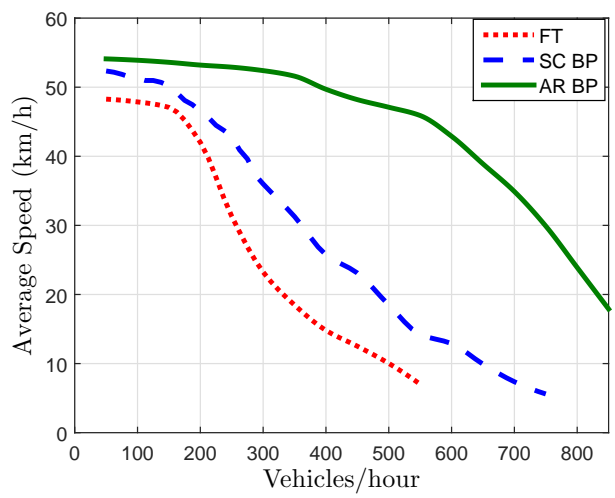

Fig. 6. Average vehicle speeds computed over a 2 hours simulation time period as a function of the vehicle input arrival rate under different methods.

always highest under AR-BP. This implies that the vehicles travel longer distances on average to reach their destinations under AR-BP, especially when the vehicle arrival rates are low. Under SC-BP and FT, a path for every vehicle from its origin to its destination is pre-defined, whereas in AR$\mathrm{BP}$ a next hop route is chosen at every intersection. When the network is under-saturated, the pressure terms (queue backlog differences) are very low and a vehicle may traverse several links before arriving its destination, thus taking a route that is unnecessarily long. However, it is this adaptive routing that forces the vehicles to distribute in the network more uniformly and thus reduces congestion queue lengths when the network is heavily loaded. In a saturated network, although vehicles may follow a longer route on average under AR-BP, the travel time is significantly lower on average compared to the fixed routing methods as shown in Fig. 5. This reduction in average travel time happens due to the smaller queue lengths in the network as observed in Fig. 4.

Next we investigate the network throughput in terms of the number of vehicles exiting the network (number of completed trips) under different signal control methods. In Fig. 7, we plot the total number vehicles that exit the given network in two hours when the traffic is continuously arriving at a fixed rate. Interestingly, FT provides higher throughput 


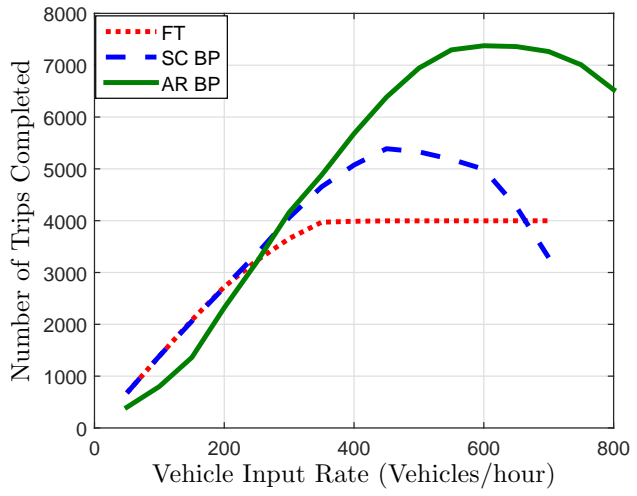

Fig. 7. Total number of vehicles that exit the network, i.e., reach their destinations during a 2 hour simulation period under different methods.

than SC-BP at very high input traffic load, despite the fact that FT always gives rise to a higher time averaged queue length than SC-BP according to Fig. 4. This happens due to the fact that when back-pressure schemes are employed over a network with finite length links, some links can experience deadlock situation, as observed in [14]. Deadlocks make the controllers non-work conserving and may cause congestion propagation to other links in a network. The deadlocks occur at very high traffic loads depending on the network topology and especially when there is a significant mismatch between lengths (or capacities) of adjacent links. Note that our simulated network is quite asymmetric in terms of lengths of different links and therefore it is also more susceptible to deadlocks. One way of resolving deadlocks under SC-BP is to use normalized pressure functions [14]. Interestingly, AR$\mathrm{BP}$ is robust against deadlocks because its control decisions are based on virtual queues that keep growing irrespective of the lengths (capacities) of their corresponding links.

\section{Conclusions and Future Directions}

We proposed a decentralized multi-commodity based back-pressure signal control and adaptive route control method (AR-BP). The performance of AR-BP was compared with the existing single-commodity based back-pressure traffic control method (SC-BP) and a fixed time schedule controller (FT). We observed that AR-BP significantly outperforms SC-BP and FT in terms of average queue lengths, average vehicle travel time, and the number of trips completed, especially when the traffic demand is high. In SC$\mathrm{BP}$ and FT, all vehicles follow fixed routes. Fixed routing is not appropriate when a network is heavily loaded with vehicles, whereas AR-BP is able to distribute vehicles more uniformly across the network, thereby significantly improving congestion, throughput, and travel times (on average). For low load situations, AR-BP may lead to unnecessarily long routes for some vehicles, giving very high travel times on average. Another important feature of AR-BP is that it is more robust to deadlock situations that SC-BP, due to the use of virtual queues.

In order to reduce travel time in low load situations, one can include bias terms in the calculation of queue backlog in the back-pressure schemes as done in wireless networks [19]. This will be a subject of our future research. Another interesting direction is to investigate AR-BP scheme subject to uncertain and delayed queue information.

\section{REFERENCES}

[1] H. Frumkin, "Urban sprawl and public health." Public Health Reports, vol. 117, no. 3, pp. 201-217, 2002.

[2] N. H. Gartner, J. D. C. Little, and H. Gabbay, "Optimization of traffic signal settings by mixed-integer linear programming," Transportation Science, vol. 9, no. 4, pp. 344-363, 1975.

[3] P. B. Hunt, D. I. Robertson, R. D. Bretherton, and R. I. Winton, "SCOOT - a traffic responsive method of coordinating signals," TRL Laboratory Report 1014, Tech. Rep., 1981.

[4] V. Mauro and C. D. Taranto, "UTOPIA," in Control, Computers, Communications in Transportation Research, J. P. Perrin, Ed. Pergamon, Oxford, 1990, pp. 245-252.

[5] P. R. Lowrie, "The Sydney co-ordinated adaptive traffic system principles, methodology, algorithms," in International Conference on Road Traffic Signaling, 1982, pp. 67-70.

[6] C. Diakaki, M. Papageorgiou, and K. Aboudolas, "A multivariable regulator approach to traffic-responsive network-wide signal control," Control Engineering Practice, vol. 10, no. 2, pp. 183 - 195, 2002.

[7] L. B. de Oliveira and E. Camponogara, "Multi-agent model predictive control of signaling split in urban traffic networks," Transportation Research Part C: Emerging Technologies, vol. 18, no. 1, pp. 120-139, Feb. 2010.

[8] H. Ezawa and N. Mukai, "Adaptive traffic signal control based on vehicle route sharing by wireless communication," in KnowledgeBased and Intelligent Information and Engineering Systems, ser. Lecture Notes in Computer Science, R. Setchi, I. Jordanov, R. Howlett, and L. Jain, Eds. Springer Berlin Heidelberg, 2010, vol. 6279, pp. 280-289.

[9] T. Tettamanti, T. Luspay, B. Kulcsár, T. Peni, and I. Varga, "Robust control for urban road traffic networks," IEEE Transactions on Intelligent Transportation Systems, vol. 15, no. 1, pp. 385-398, Feb. 2014

[10] S. Lämmer and D. Helbing, "Self-control of traffic lights and vehicle flows in urban road networks," Journal of Statistical Physics, April 2008

[11] T. Wongpiromsarn, T. Uthaicharoenpong, Y. Wang, E. Frazzoli, and D. Wang, "Distributed traffic signal control for maximum network throughput," in IEEE Conference on Intelligent Transportation Systems (ITSC), Sept 2012, pp. 588-595.

[12] P. Varaiya, "The max-pressure controller for arbitrary networks of signalized intersections," in Advances in Dynamic Network Modeling in Complex Transportation Systems, ser. Complex Networks and Dynamic Systems, S. V. Ukkusuri and K. Ozbay, Eds. Springer New York, 2013, vol. 2, pp. 27-66.

[13] J. Gregoire, E. Frazzoli, A. de La Fortelle, and T. Wongpiromsarn, "Back-pressure traffic signal control with unknown routing rates," in IFAC World Congress, 2014.

[14] J. Gregoire, X. Qian, E. Frazzoli, A. de La Fortelle, and T. Wongpiromsarn, "Capacity-aware back-pressure traffic signal control," IEEE Tranactions on Control of Networked Systems, Submitted 2014. [Online]. Available: http://arxiv.org/abs/1309.6484

[15] L. Tassiulas and A. Ephremides, "Stability properties of constrained queueing systems and scheduling policies for maximum throughput in multihop radio networks," IEEE Transaction on Automatic Control, vol. 37, no. 12, pp. 1936-1948, 1992.

[16] E. Athanasopoulou, L. X. Bui, T. Ji, R. Srikant, and A. Stolyar, "Backpressure-based packet-by-packet adaptive routing in communication networks," IEEE/ACM Transactions on Networking, vol. 21, no. 1, pp. 244-257, Feb. 2013.

[17] L. B. Le, E. Modiano, and N. B. Shroff, "Optimal control of wireless networks with finite buffers," IEEE/ACM Transactions on Networking, vol. 20, no. 4, Aug. 2012.

[18] M. Fellendorf, "VISSIM: a microscopic simulation tool to evaluate actuated signal control including bus priority," in 64th ITE Annиal Meeting, 1994.

[19] M. J. Neely, "Dynamic power allocation and routing for satellite and wireless networks with time varying channels," Ph.D. dissertation, Massachusetts institute of Technology, LIDS, 2003. 